\title{
I-Dizisel Hausdorff Uzaylar
}

\section{I-Sequentially Hausdorff Spaces}

\section{Hürmet Fulya AKIZ*}

Bozok Üniversitesi, Fen Edebiyat Fakültesi, Matematik Bölümü, 66900, Yozgat

• Geliş tarihi / Received: 03.04.2019 • Düzeltilerek geliş tarihi / Received in revised form: $22.05 .2019 \quad$ • Kabul tarihi / Accepted: 03.06 .2019

\section{$\ddot{O} z$}

Bu çalışmada öncelikle $I$-dizisel sürekli, $I$-dizisel açık ve $I$-dizisel kapalı fonksiyonlar tanıtılmıștır. Daha sonra, Hausdorff uzaylardan daha geniş bir yapı olan I-dizisel Hausdorff uzayların tanımı ve bazı özellikleri verilmiş̧ir.

Anahtar kelimeler: Hausdorff uzaylar, $I$-dizisel açık küme, $I$-dizisel kapalı küme

\begin{abstract}
In this study, we first introduce the notions of I-sequentially continuous, I-sequentially open and I-sequentially closed functions. We also give the definition and some properties of a I-sequentially Hausdorff space, which is a wider structure than a Hausdorff space.
\end{abstract}

Keywords: Hausdorff spaces, I-sequentially open set, I-sequentially closed set

* Hürmet Fulya AKIZ; fulya.gencel@bozok.edu.tr; Tel:(0354) 24210 21-2578; orcid.org/0000-0002-8547-2175 


\section{Giriş}

Reel sayılar kümesindeki yakınsaklık kavramı istatistiksel yakınsaklık kavramına genişletilmiştir (Fast, 1951; Schoenberg, 1959). NN, doğal sayılar kümesinin bir alt kümesi $K$ olmak üzere, $\left|K_{n}\right|$, $K_{n}=\{k \in K: k \leq n\}$ kümesinin eleman sayısını göstersin. Bu durumda

$d(K)=\lim _{n \rightarrow \infty} \frac{\left|K_{n}\right|}{n}$

limiti mevcut ise bu limit değeri $K$ kümesinin yoğunluğu olarak tanımlanır (Niven, 1980; Halberstem ve Roth, 1993).

$\left(x_{n}\right)$ reel terimli bir dizi olmak üzere her $\mathcal{E}>0$ için

$K(\varepsilon)=\left\{k \in K:\left|x_{k}-l\right| \geq \mathcal{E}\right\}$

kümesinin doğal yoğunluğu sıfıra eşit ise $\left(x_{n}\right)$ dizisi $l$ değerine istatistiksel yakınsaktır denir (Fast, 1951; Schoenberg, 1959).

Daha önce bir topolojik uzayda dizisel açık ve dizisel kapalı kümeler yardımıyla birçok özellik yeniden ele alınmıştır. $\mathrm{Bu}$ tanımlar genelleştirilerek $G$-yakınsaklık kavramı ile topolojik uzaylarda $G$-dizisel açık küme, $G$-dizisel kapalı küme, $G$-dizisel süreklilik, $G$-dizisel irtibatlılık gibi konular incelenmiştir (Çakallı, 2011, 2012; Çakallı ve Mucuk, 2013; Mucuk ve Şahan, 2014). Bu çalışmalarda elde edilen sonuçlar, birinci sayılabilirlik aksiyomunu sağlayan Hausdorff topolojik gruplar içindir.

Reel sayılardaki istatistiksel yakınsaklık kavramı bir $I$ ideali yardımıyla $I$-yakınsaklık kavramına genişletilebilir. Bu alanda istatistiksel yakınsaklık ve $I$-yakınsaklık kavramları ile ilgili tanım ve teoremler verilmiştir (Kostyrko vd., 2001, 2005).

Pal (2014) ise bir $I$ ideali yardımıyla $I$-dizisel açık ve $I$-dizisel kapalı kümeleri tanımlamışırır. Ayrıca topolojik uzaylarda I-dizisel kompaktlık kavramı ve bazı özellikleri verilmiştir (Banerjee ve Banerjee, 2015).

Dizisel açık kümeler yardımıyla yapılan dizisel Hausdorff uzay tanımı, bu uzayların Hausdorff uzaylarla ilişkisi ve bazı özellikleri Akız ve Koçak (2019) da verilmiştir.

Bu çalışmada, ilk olarak $I$-dizisel açık ve $I$-dizisel kapalı fonksiyonlar tanıtılmıştır. Daha sonra $I$ dizisel açık kümeler yardımıyla, Hausdorff uzay kavramından daha geniş olan I-dizisel Hausdorff uzayların tanımı ve bazı özellikleri verilmiştir.

\section{Temel Tanım ve Teoremler}

2.1. Tanım: $X$ boştan farklı bir küme $I \subset 2^{X}$ sınıfı $X$ in alt kümelerinin bir sinıfı olsun. Bu durumda aşağıdaki şartlar sağlanıyor ise $I$ ya $X$ in bir ideali denir.
(i)
(ii) $A \in I, B \subset A$ ise $B \in I$ dır.

Eğer $I \neq\{\varnothing\}$ ve $X \notin I$ ise $I$ ya aşikar olmayan ideal denir. Eğer $I$ her tek nokta kümesini içeriyor ise $I$ ya uygun ideal denir (Kostyrko vd., 2001).

Bundan sonra $X$ bir topolojik uzay ve $I$ da $\mathbb{N}$ doğal sayılar kümesinin bir aşikar olmayan ideali olarak kabul edilecektir.

2.2. Tanım: $\left(x_{n}\right), X$ de bir dizi, $x \in X$ ve $I \subset 2^{\mathbb{N}}$ aşikar olmayan bir ideal olsun. Eğer $x$ in her $U$ açık komşuluğu için $\left\{n \in \mathbb{N}: x_{n} \notin U\right\} \in I$ ise $\left(x_{n}\right)$ dizisi $x$ elemanına $I$-yakınsaktır denir (Lahiri ve Das, 2005).

Bu durumda $x$ noktasina $\left(x_{n}\right)$ dizisinin $I$-limiti denir ve $I-\lim x_{n}=x$ olarak gösterilir.

2.3.Not: Eğer $I$ bir uygun ideal ise bu durumda yakınsaklık kavramı I-yakınsaklığı gerektirir. Eğer $I$ ideali yalnızca sonlu küme içeriyor ise bu ifadenin tersi de doğrudur.

2.4. Tanım: $O \subseteq X$ ve $I \subset 2^{\mathbb{N}}$ aşikar olmayan bir ideal olsun. Eğer $I$-limiti $O$ da iken kendisi $O^{c}$ de olan bir dizi yoksa $O$ kümesine $I$-dizisel açık küme denir (Pal, 2014).

2.5. Tanım: $O \subseteq X$ ve $I \subset 2^{\mathbb{N}}$ aşikar olmayan bir ideal olsun. $\left(x_{n}\right), K$ da bir dizi ve $I-\lim x_{n}=x$ iken $x \in K$ ise $K$ ya $I$-dizisel kapalı küme denir (Pal, 2014).

2.6. Önerme: Her açık küme I-dizisel açıktır.

İspat: $A \subseteq X$ açik bir küme, $\left(x_{n}\right)$ dizisi $A^{c}$ da bir dizi ve $y \in A$ olsun. Bu durumda $y \in U \subseteq A$ olacak şekilde bir $U$ açık komşuluğu vardır. $U$ kümesi $\left(x_{n}\right)$ dizisinin hiçbir terimini içermez. Dolayısıyla $\left(x_{n}\right)$ dizisi y noktasina $I$ - yakınsak değildir. $\mathrm{O}$ halde $A$ kümesi $I$-dizisel açıktır.

$\mathrm{Bu}$ önermenin tersinin doğru olmadığ 1 aşağıdaki örnekte görülür. 
2.7. Örnek: $\mathbb{R}$, reel sayılar kümesi üzerinde $\tau=\left\{G \subseteq \mathbb{R}: G^{c}\right.$ sayllabilir $\} \cup\{\varnothing\} \quad$ tümleyeni sayılabilir topolojisini göz önüne alalım. $\mathrm{Bu}$ topolojiye göre bir $A \subseteq \mathbb{R}$ kapalıdır ancak ve ancak ya $A=\mathbb{R}$ ya da $A$ sayılabilirdir. $\left(x_{n}\right), I-$ limiti $y$ olan bir dizi olsun. Bu durumda $(\mathbb{R} \backslash$ $\left.\left\{x_{n}: n \in \mathbb{N}\right\}\right) \cup\{y\}$ kümesi $y$ nin bir açık komşuluğu olup dizinin sonsuz terimini içermelidir. $\mathrm{Bu}$ topolojiye göre bir dizinin yakınsak olması için belli bir indisten sonra terimlerinin sabit olmas1 yani $x_{n}=y$ olmas1 gerekir. Dolayısıyla $A$ daki bir dizi ancak $A$ nın bir elemanına yakınsayabilir ve $A$ kümesi $I$ dizisel açiktır. Yani bu topolojiye göre her küme I-dizisel açıktır. Fakat her alt küme açık değildir.

\section{I-Dizisel Hausdorff Uzaylar ve Özellikleri}

Bu bölümde, ilk olarak $I$-dizisel açık komşuluk, $I$ dizisel sürekli fonksiyon, $I$-dizisel açık ve $I$-dizisel kapalı fonksiyon kavramları tanitılacaktır. Daha sonra Hausdorff uzay kavramından daha geniş olan I-dizisel Hausdorff uzay tanımı ve onun baz1 özellikleri verilecektir.

3.1. Tanım: $X$ bir topolojik uzay ve $I \subset 2^{\mathbb{N}}$ aşikar olmayan bir ideal olsun. Eğer bir $a \in X$ için $a \in O \subseteq A$ olacak şekilde $I$-dizisel açık olan bir $O$ kümesi varsa $A$ ya $a$ elemanının bir $I$-dizisel açık komşuluğu denir.

3.2. Teorem: $X$ bir topolojik uzay ve $I \subset 2^{\mathbb{N}}$ aşikar olmayan bir ideal olsun. $X$ de $I$-dizisel kapalı olan bir kümenin tümleyeni $I$-dizisel açıktır.

İspat: $F \subseteq X$ kümesi $I$-dizisel kapalı olsun. $F^{c}$ nin $I$-dizisel açık olduğunu göstermek gerekir. $x \in F^{c}$ ve $\left(x_{n}\right), F$ de bir dizi ve $I-\lim x_{n}=x$ olsun. $F$, I-dizisel kapalı olduğundan $x \in F$ olmalıdır. $\mathrm{Bu}$ ise çelişkidir. $\mathrm{O}$ halde $F^{c}$ deki bir elemana $I-$ yakınsak olan ve $F$ de bulunan bir dizi yoktur. $F^{c}$, I-dizisel açıktır.

3.3. Tanım: $X$ ve $Y$ topolojik uzaylar, $f: X \rightarrow Y$ bir fonksiyon ve $x \in X$ olsun. Eğer $I-\lim x_{n}=$ $x$ olacak şekildeki her $\left(x_{n}\right)$ dizisi için $I-$ $\lim f\left(x_{n}\right)=f(x)$ ise $f$ fonksiyonuna $I$-dizisel süreklidir denir.

3.4. Önerme: Sürekli bir fonksiyon I-dizisel süreklidir.

İspat: $f: X \rightarrow Y$ fonksiyonu sürekli olsun. $\left(x_{n}\right), X$ de bir dizi ve $I-\lim \left(x_{n}\right)=x$ olsun. $\mathrm{Bu}$ durumda $\quad I-\lim f\left(x_{n}\right)=f(x) \quad$ olduğunu göstermek gerekir. $f$ sürekli olduğundan, $f(x)$ in her $V$ açık komşuluğu için $x$ elemanının $f(U) \subseteq$ $V$ olacak şekilde bir $U$ açık komşuluğu vardır. $I-\lim \left(x_{n}\right)=x$ olduğundan $\left\{n \in \mathbb{N}: x_{n} \notin U\right\} \in I$ dır. Buradan $f(x)$ in $V$ açık komşuluğu için $\left\{n \in \mathbb{N}: f\left(x_{n)} \notin f(U) \subseteq V\right\} \in I \quad\right.$ olup $\quad I-$ $\lim f\left(x_{n}\right)=f(x)$ dir.

$\mathrm{Bu}$ önermenin tersinin doğru olmadığına dair aşağıdaki örnek verilebilir.

3.5. Örnek: $\mathbb{R}$, reel sayılar kümesi üzerinde $U$ alış1 1 ıış topolojisi ve

$\tau=\left\{G \subseteq \mathbb{R}: G^{c}\right.$ sayllabilir $\} \cup\{\varnothing\}$

tümleyeni sayılabilir topolojisini göz önüne alınsin.

$f:(\mathbb{R}, \boldsymbol{\tau}) \rightarrow(\mathbb{R}, U), x \mapsto x$

fonksiyonu $I$-dizisel sürekli olmasına rağmen sürekli değildir.

3.6. Tanım: $X$ ve $Y$ topolojik uzaylar, $f: X \rightarrow Y$ bir fonksiyon olsun. Eğer $X$ deki her $I$-dizisel açık kümenin görüntüsü $Y$ de $I$-dizisel açık ise $f$ fonksiyonuna $I$-dizisel açık fonksiyon denir.

3.7. Tanım: $X$ ve $Y$ topolojik uzaylar, $f: X \rightarrow Y$ bir fonksiyon olsun. Eğer $X$ deki her I-dizisel kapalı kümenin görüntüsü $Y$ de $I$-dizisel kapalı ise $f$ fonksiyonuna $I$-dizisel kapalı fonksiyon denir.

3.8. Tanım: $X$ bir topolojik uzay olsun. Farklı her nokta çifti $x, y \in X$ için, $x \in O, y \in P$ ve $O \cap P=$ $\varnothing$ olacak şekilde $I$-dizisel açik olan $O$ ve $P$ kümeleri varsa $X$ uzayına $I$-dizisel Hausdorff uzay denir.

3.9. Örnek: $\mathbb{R}$, reel sayılar kümesi üzerinde $\tau=\left\{G \subseteq \mathbb{R}: G^{c}\right.$ sayılabilir $\} \cup\{\varnothing\} \quad$ tümleyeni sayılabilir topolojisine göre I-dizisel Hausdorff uzaydır. Bu uzayda her küme I-dizisel açık olduğundan $O=\{x\}$ ve $P=\{y\} \quad$ kümeleri sirasiyla $x$ ve $y$ yi içeren $I$-dizisel açık kümelerdir. Aynı zamanda $O \cap P=\varnothing$ olduğundan bu uzay $I$ dizisel Hausdorfftur.

3.10. Örnek: En az iki elemanlı bir $X$ kümesi, üzerinde tanımlanan diskre(ayrık) topolojiye göre I-dizisel Hausdorfftur.

3.11. Örnek: $X$ boştan farklı bir küme olmak üzere, üzerindeki indiskre(ayrı olmayan) topolojiye göre I-dizisel Hausdorff değildir. Çünkü bu uzayda $I$-dizisel açık olan tek küme $X$ dir. 
3.12. Örnek: $\mathbb{R}$, reel sayılar kümesi, üzerindeki alışılmış topolojiye göre I-dizisel Hausdorfftur.

3.13. Önerme: $\operatorname{Bir} X$ topolojik uzayı Hausdorff ise I-dizisel Hausdorfftur.

Ispat: $X$ topolojik uzayı Hausdorff olduğundan farklı her nokta çifti $x, y \in X$ için, $x \in O, y \in P$ ve $O \cap P=\emptyset$ olacak şekilde açık olan $O$ ve $P$ kümeleri vardır. Her açık küme aynı zamanda $I$ dizisel açık olduğundan $O$ ve $P$ kümeleri sırasıyla $x$ ve $y$ yi içeren $I$-dizisel açık kümelerdir.

Fakat bu ifadenin tersinin genelde doğru olmadığ aşağıdaki örnekten anlaşılabilir.

3.14. Örnek: $\mathbb{R}$, reel sayılar kümesi, tümleyeni sayılabilir topolojiye göre I-dizisel Hausdorfftur. Fakat bu uzay Hausdorff değildir.

3.15. Önerme: Her metrik uzay I-dizisel Hausdorfftur.

İspat: Her metrik uzay Hausdorff olup Önerme 3.11 den I-dizisel Hausdorftur.

I-dizisel Hausdorff olan bir uzayın bir alt uzayıla ilgili olan teoremi vermeden önce aşağıdaki önerme ispatlanacaktır.

3.16. Önerme: $X$ bir topolojik uzay ve $A \subseteq X$ olsun. Bir $O \subseteq X$ kümesi $I$-dizisel açık ise $A \cap O$ kümesi de $A$ üzerindeki alt topolojiye göre $I$ dizisel açıktır.

İspat: $A$ kümesi, üzerindeki alt topoloji ile göz önüne alınsın. $a \in A \cap O$ ve $\left(x_{n}\right)$ dizisi $(A \cap$ $O)^{c}$ de bir dizi olsun. Bu durumda $\left(x_{n}\right), A^{c} \cap O^{c}$ de bir dizidir. $\left(x_{n}\right)$ in $A$ da bir dizi olduğundan, bu gösterir ki dizinin terimleri $O^{c}$ de bulunmaz. $O$, $I$-dizisel açık olduğundan $\left(x_{n}\right)$ dizisi $a$ elemanına $I$-yakınsak olamaz. O halde $A \cap O$ kümesi $I$ dizisel açıktır.

3.17. Önerme: $X$ uzay1 $I$-dizisel Hausdorff uzay ve $A \subseteq X$ olsun. $A$ kümesi de üzerindeki alt topoloji ile beraber $I$-dizisel Hausdorff uzaydır.

Ispat: $a, b \in A \subseteq X$ olsun. $X$ uzayı I-dizisel Hausdorff olduğundan $a \in O, b \in P$ ve $O \cap P=$ $\varnothing$ olacak şekilde $I$-dizisel açık olan $O$ ve $P$ kümeleri vardır. Önerme 3.14. den $A \cap O$ ve $A \cap P$ kümeleri sirasiyla $a$ ve $b$ yi kapsayan $I$ dizisel açık kümelerdir. $(A \cap O) \cap(A \cap P)=\varnothing$ olduğundan $A$ üzerindeki alt topoloji $I$-dizisel Hausdorfftur.
Hausdorff uzaylarda iyi bilinen bir sonuç I-dizisel Hausdorff uzaylar için aşağıdaki şekilde gösterilebilir.

3.18. Teorem: $I$-dizisel Hausdorff bir uzayda $I$ yakınsak bir $\left(x_{n}\right)$ dizisinin $I$-limiti tektir.

İspat: $\left(x_{n}\right)$ dizisi için $I-\lim x_{n}=x$ ve $I-$ $\lim x_{n}=y \quad$ olacak şekilde farklı $x, y \in X$ elemanlar1 olsun. $X$ uzayı I-dizisel Hausdorff olduğundan $x$ ve $y$ yi içeren $O$ ve $P$ ayrık $I$-dizisel açık kümeleri vardır. $I-\lim x_{n}=x$ olduğundan $\left\{n \in \mathbb{N}: x_{n} \notin O\right\} \in I$ olup $\left(x_{n}\right)$ dizisisinin ancak sonlu terimi $O$ nun dıșındadır. Benzer şekilde $I-\lim x_{n}=y$ olduğundan $\left\{n \in \mathbb{N}: x_{n} \notin P\right\} \in I$ olup $\left(x_{n}\right)$ dizisisinin ancak sonlu terimi $P$ nun dışındadır. Bu durumda $O \cap P \neq \varnothing$ olur. $\mathrm{Bu}$ ise kabuldeki ifade ile çelişir. O halde $x=y$ dir.

Hausdorff uzay olma kavramı bir topolojik özelliktir (Mucuk, 2010). I-dizisel Hausdorff olma kavramının da belli şartlar altında korunduğu aşağıda teoremde görülebilir.

3.19. Teorem: $X$ ve $Y$ topolojik uzaylar ve $f: X \rightarrow Y$ fonksiyonu sürekli, birebir, örten ve $I$ dizisel açı olsun. Bu durumda $X$ uzayı $I$-dizisel Hausdorff ise $Y$ uzayı da $I$-dizisel Hausdorfftur.

İspat: Farklı nokta çifti $y_{1}, y_{2} \in Y$ ele alınsın. $f$ fonksiyonu birebir ve örten olduğundan $f\left(x_{1}\right)=$ $y_{1}$ ve $f\left(x_{2}\right)=y_{2}$ olacak şekilde $x_{1}, x_{2} \in X$ vardır ve $x_{1} \neq x_{2}$ dir. $X$ uzay1 $I$-dizisel Hausdorff olduğundan, $x_{1} \in G, x_{2} \in H$ olacak şekilde ayrık $G$ ve $H \quad I$-dizisel açık komşuluklar vardır. $f$ fonksiyonu $I$-dizisel açı olduğundan $f(G)$ ve $f(H)$ sirasiyla $y_{1}$ ve $y_{2}$ nin $I$-dizisel açık komşuluklarıdır. Buradan $\quad f(G) \cap f(H)=$ $f(G \cap H)=\varnothing$

3.20. Teorem: Bir $X$ topolojik uzayında aşağıdaki ifadeler denktir:

(i) $X$ uzayı $I$-dizisel Hausdorfftur.

(ii) $\Delta X=\{(x, x): x \in X\}$ diyagonal kümesi $X \times X$ in $I$-dizisel kapalı bir alt cümlesidir.

(iii) $\Delta: X \rightarrow X \times X, x \rightarrow(x, x) \quad$ diyagonal fonksiyonu $I$-dizisel kapalıdır.

İspat: (i) $\Rightarrow$ (ii) sağlandığ 1 gösterilsin. $X$ uzayı $I-$ dizisel Hausdorff uzay olsun. $\Delta X$ kümesinin $I$ dizisel kapalı olduğunu göstermek için $(\Delta X)^{c}$ kümesinin I-dizisel açı olduğu gösterilmelidir. $(x, y) \in(\Delta X)^{c}$ olsun. $x \neq y$ ve $X$ uzayı $I$-dizisel Hausdorff olduğundan sirasıyla $x$ ve $y$ yi içeren $O$ ve $P$ ayrık $I$-dizisel açık kümeleri vardır. $O, I$ - 
dizisel açık olduğundan $O^{c}$ da $x$ e $I$-yakınsak olan bir dizi yoktur. Benzer şekilde $P, I$-dizisel açık olduğundan $P^{c}$ de $y$ elemanına $I$-yakınsak olan bir dizi yoktur. $O \times P \subseteq(\Delta X)^{c}$ kartezyen çarpım kümesi de $(x, y)$ nin $I$-dizisel açık komşuluğudur. $\mathrm{O}$ halde $\Delta X$ de $(x, y)$ ye yakınsayan bir dizi yoktur.

(ii) $\Rightarrow$ (iii) sağlandığ 1 gösterilsin. $\Delta X \subseteq X \times X \quad I$ dizisel kapalı olsun.

\section{$\Delta: X \rightarrow X \times X, x \rightarrow(x, x)$}

fonksiyonunun $I$-dizisel kapalı olduğunu göstermek için bir $K \subseteq X \quad I$-dizisel kapalı cümlesini göz önüne alınsın. $\Delta(K)$ nın $I$-dizisel kapalı olduğunu göstermek için $\Delta(K)^{c}$ nin $I$ dizisel açık olduğunu göstermek yeterli olacaktır.

Eğer $x \neq y$ ise $(x, y) \in(\Delta X)^{c}$ olur. $X$ uzayı $I$ dizisel Hausdorff olduğundan $x$ ve $y$ yi içeren $O$ ve $P$ ayrık $I$-dizisel açık kümeleri vardır. $O$ kümesi $I$-dizisel açık olduğundan $O^{c}$ de $x$ e $I$ yakınsak olan bir dizi yoktur. Benzer şekilde. $P$ kümesi $I$-dizisel açık olduğundan $P^{c}$ de $y$ ye $I$ yakınsak olan bir dizi yoktur. $O \times P \subseteq(\Delta X)^{c} \subseteq$ $\Delta(K)^{c}$ olduğundan $\Delta(K)$ da $(x, y)$ ye $I$-yakınsak olan bir dizi yoktur. $\mathrm{O}$ halde $\Delta(K)^{c}$ nin $I$-dizisel açıktır.

Eğer $x=y$ ve $x \notin K$ olsun. $K, I$-dizisel kapalı ise $K^{c}$ nin $I$-dizisel açık olduğunu göstermek yeterlidir. $x \in K^{c}$ için $K$ da $x$ e $I$-yakınsak olan bir dizi yoktur. O halde $\Delta(K)^{c}$ de $(x, x)$ e $I$ yakınsak olan bir dizi yoktur. Yani $\Delta(K)^{c}$ açıktır.

(iii) $\Rightarrow$ (i) sağlandığı gösterilsin.

$\Delta: X \rightarrow X \times X, x \rightarrow(x, x)$

fonksiyonu $I$-dizisel kapalı olsun. Farklı nokta çifti $x, y \in X$ alalım. Bu durumda $(x, y) \in(\Delta X)^{c}$ olur. $(\Delta X)^{c}, I$-dizisel açık olduğundan $(x, y)$ ye $I$ yakinsak olan ve $\Delta X$ de bulunan bir dizi yoktur. $\mathrm{Bu}$ durumda $O \times P \subseteq(\Delta X)^{c}$ olacak şekilde sirasiyla $x$ ve $y$ yi içeren $I$-dizisel açık komşuluklar vardır. $(\Delta X)^{c}$ de $O$ ve $P$ nin ortak elemanı bulunmadığından $O \cap P=\varnothing$ dur. $O$ halde $X$ uzay $I$-dizisel Hausdorff olur.

\section{İspat: 4. Sonuç ve Öneriler}

Topolojik ve kalitsal bir özellik olan Hausdorff uzay kavramına benzer olarak, I-dizisel açık kümeler yardımıyla daha geniş bir yapı olan $I$ dizisel Hausdorff uzaylar kavramı tanımlanmıştır. $\mathrm{Bu}$ durumda Pal (2014) de tanımlanan I-dizisel Topolojik uzaylar göz önüne alınırsa, bu iki kavramın birbirine denk olduğu görülür. $I$-dizisel Hausdorffluk da sürekli fonksiyonlar altında korunan bir özelliktir. Ayrıca Hausdorff uzaylar gibi kalıtsal özelliğe de sahiptir. Bu çalışmada elde edilen sonuçlar, diğer ayırma aksiyomları için de $I$-yakınsaklık kavramı yardımıyla yeniden ele alınıp incelenebilir.

\section{Kaynaklar}

Akız, H. F. ve Koçak, L., 2019. Sequentially Hausdorff and Full Sequentially Hausdorff Spaces, Commun. Fac. Sci. Univ. Ank. Series A1, 68 (2), 1724-1732.

Banerjee, A. K. ve Banerjee, A., 2015. A Note On IConvergence and $I^{*}$-Convergence of Sequences and Nets In Topological Spaces, Matematicki Vesnik, 67(3), 212-221.

Çakallı, H. ve Mucuk, O., 2013. On connectedness via a sequential method, Revista de la Union Matematica Argentina, 54 (2), 101-109.

Çakallı, H., 2011. On G-continuity, Comput. Math. Appl., 61, 313-318.

Çakallı, H., 2012. Sequential definitions of connectedness, Appl. Math. Lett., 25, 461-465.

Fast, H., 1951. Sur la convegence statistique, Collog. Math. 2, 241-244.

Halberstem, H. ve Roth, K. F., 1993. Sequences, Springer, New York.

Kostyrko, P., Mačaj, M., Šalát, T. ve Sleziak, M., 2005. I-convergence and a extermal I-limit points, Math. Slovaca, 55 (4),443-464.

Kostyrko, P., Šalát, T. ve Wilczynski, W., 2001. Iconvergence, Real Analysis Exch. 26 (2), 669686.

Lahiri, B. K. ve Das, P., 2005. $I$ and $I^{*}$-convergence in topological spaces, Math. Bohemica, 130 (2), 153-160.

Mucuk O. ve Şahan T., 2014. On G-Sequential Continuity, Filomat, vol.28, 1181-1189.

Mucuk O., 2010. Topoloji ve Kategori, Nobel Yayınları, Ankara.

Niven, I. ve Zuckerman, H. S., 1980. An introduction to the theory of numbers. 4th Ed., John Wiley, New York.

Pal, S. K., 2014. I-Sequential Topological Spaces, Applied Mathematics E-notes, 14, 236-241.

Schoenberg, I. J., 1959. The integrability of certain function and related summability methods. Am. Math. Mon. 66, 361-375. 